\title{
Towards Mobile Blended Interaction Fostering Critical Thinking
}

\section{Adrian Holzer}

EPFL

1015 Lausanne, Switzerland

adrian.holzer@epfl.ch
Samuel Bendahan

EPFL

1015 Lausanne, Switzerland samuel.bendahan@epfl.ch

\author{
Sten Govaerts \\ EPFL \\ 1015 Lausanne, Switzerland \\ sten.govaerts@epfl.ch \\ Denis Gillet \\ EPFL \\ 1015 Lausanne, Switzerland \\ denis.gillet@epfl.ch
}

Permission to make digital or hard copies of part or all of this work for personal or classroom use is granted without fee provided that copies are not made or distributed for profit or commercial advantage and that copies bear this notice of this work must be honored. For all other uses, contact the Owner/Author. Copyright is held by the owner/author(s). MobileHCl '15 Adjunct August Copyright is held by the owner/author(s). Mobi 14C1'15/Adjunt, August http://dx.doi.org/10.1145/2786567.2793695

\author{
Abstract \\ Critical thinking is a fundamental skill that all citizens \\ should have. Unfortunately, while beliefs in unfounded \\ claims are pervasive, teaching critical skill is a strenuous \\ task. Digital mobile interaction could potentially support \\ face-to-face teaching to foster critical thinking skills. In \\ this paper, we present a preliminary version of the \\ BaloneyMeter, a mobile app that provides support for \\ teaching critical thinking skills. We report on a case study \\ in a class of 150 students and present an open research \\ question that we plan to address in subsequent work.

\section{Author Keywords} \\ Critical thinking; $\mathrm{HCl}$; Paranormal beliefs; Baloney \\ detection kit; Blended learning

\section{ACM Classification Keywords} \\ H.5.2 [Information Interfaces and Presentation]: User \\ Interfaces - Interaction styles

\section{Introduction} \\ In the age of social media, with the amount of data \\ available on the internet doubling every 2 years [7], it is \\ crucial for citizens to be able to correclty assess claims \\ that they are bombarded with every day. Indeed, with the \\ advent of the Web there has been an increase in our \\ exposure to unfounded claims (paranormal,
}


pseudo-scientific, conspiracy theories, rumors) [8] and research found that for every two additional rumors an individual hears, the average number of rumors believed increases by about one [8]. On the positive side, the Web has also allowed to increase the number of rebuttals. Unfortunately, rebuttals are only half as effective as the exposure to rumors [8]. Furthermore, with the rise of social media, sharing of rumors has become easier than ever and (mis)information coming directly from friends is particularily persuasive [8]. Simply learning if a claim is trustworthy is not enough since it does not inform individuals on future claims, it is thus essential to be able to personally assess the validity of claims through critical thinking [11]. This is why the famous science educator and skeptic, the late Carl Sagan, had laid out a series of questions to guide reflection in what he called the Baloney Detection Kit $[15,16]$. This kit can serve as useful framework to stucture a course on critical thinking. Providing adequate designs for digital interaction supporting such courses is the focus of this paper. Indeed, even though mobile blended learning is on the rise, there is a lack research in the $\mathrm{HCl}$ literature that addresses support for teaching critical thinking.

In this paper, we aim at providing a first step to such blended mobile interaction. We first briefly discuss how unfounded beliefs arise, how they could be corrected and how the $\mathrm{HCl}$ literature has addressed the question. Then, we present the BaloneyMeter, a mobile application that presents some critical thinking questions in a playful way. Then, we disscuss a case study in a class with 150 first-year engineering students, where we created a set of critical thinking lectures using the BaloneyMeter as support. We report on the paranormal beliefs of the students and on their attitude towards the usability of the app. Finally we wrap up with an outlook on future work.

\section{Related work}

Here we review the research efforts to understand beliefs in unfounded claims (e.g., paranormal beliefs, conspiracies, rumors). We further review how the $\mathrm{HCl}$ literature linked to the topic.

A wealth of research in psychology and sociology has been interested in determining the orgins and the nature of beliefs in unfounded claims. Research shows that there is a link between these beliefs [6]. Prior research has argued that people who endorse conspiracy thinking are prone to the conjunction fallacy, by which they overestimate the likelyhood of co-occuring events [5]. Furthermore, such thinking can be linked to personality traits, such as boredom [4] or paranoia, some of which tend to be more prevalent in marginal groups in the society (e.g., women, youth, minorities, lower SES) [13]. It can also be looked at from a cultural sociology perspective and reflect a form of protest against the powerful [19]. It has also been

found that people with an analytic cognitive style are less likely to endorse paranormal beliefs, controlling for cognitive ability [14].

Research suggests that most of these beliefs are fixed quite early, ie., before higher education [1]. Furthermore, it is difficult to correct them simply by pointing out the better alternative explanation. Indeed in people who strongly hold certain unfounded beliefs, trying to correct them might even further strengthen their beliefs [10]. It is thus crucial to teach skepticism and scientific thinking early as it is most likely to exert an influence at the time of message exposure [10]. An effective method to foster science-based reasoning is to ask students to generate counter arguments for unfounded beliefs themselves [12]. The ability to cite evidence to justify ones belief is considered a primary form of scientific literacy that should 
be taught alongside scientific content [24] but is unfortunately missing even among people holding a degree in a scientific flied of study [18].

In order to provide a toolkit to evaluate claims and provide proper justification, Carl Sagan layed out his Baloney Detection Kit in the Deamon-Hauted World [15, 16].

This kit was later refined by Michael Shermer, the president of the Skeptic society [17]. This kit consists of questions that should be asked before accepting a claim. For instance questions about the quality of evidence for the claim, about possible alternative claims, and about potential fallacies in the argumentation. As mentioned above, this toolkit provides a useful framework to set up a course around critical information assessment.

Recent research suggests that beliefs in pseudoscientific claims could be reduced through online

argumentation [23]. However, understanding how to design adequate interaction to support critical thinking education in the classroom is still a largely unresolved issue. Indeed, the $\mathrm{HCl}$ literature has only rarely investigated pseudoscientific beliefs directly. For instance some authors suggested to use magic and paranormal vocabulary as metaphors for tangible user interfaces [20]. Others used a paranormal storyline to investigate how to enhance television shows by intgrating the biodata of actors [21]. One study direclty studied paranormal beliefs in the context of $\mathrm{HCl}$ and investigated the relationship between thinking styles and the use of technology as information source [2]. This study found that individuals who rely on a less analytic thinking style, and thus tend to have lower critical thinking skills [14], rely more on their smartphone for information [2]. This indicates that a mobile interface could be a good place to foster critical thinking for those who would need it the most.

\section{The BaloneyMeter}

The BaloneyMeter (see Figure 1) is a mobile app that allows users to evaluate a claim by answering a set of five questions derived from the Baloney Detection

Kit $[15,16] .{ }^{1}$ The questions are the following:

- Is the claim extraordinary? This question is intended to give the intuition that "extraordinary claims require extraordinary evidence" as was famously put by Sagan. Extraordinary claim typically cover claims that if true would require to revise much of other existing knowledge (e.g., I can levitate, star positions at birth influence personnality).

- Is the source of the claim reliable? This question aims at bringing the attention to the fact that some sources are more reliable than others, for instance systematic research reviews are usually more reliable than single scientific articles which are more usually more reliable than the opinion of a single expert.

- Can the claim be tested? This question points to the fact that if a claim can in principle neither be confirmed nor rejected, it is not worth much.

- Is there evidence for the claim? This question is a reminder that experimentation, and in particular controlled experiments, are key to assessing if a claim is true. Anecdotes on the other hand do not inform on the validity of a claim.

- Is there evidence against the claim? This last question reminds us that one should always play with different alternatives before settling for a claim or an explanation.

${ }^{1}$ The BaloneyMeter is available on the Apple App Store for free. An online version is available on www. baloneymeter. ch. 


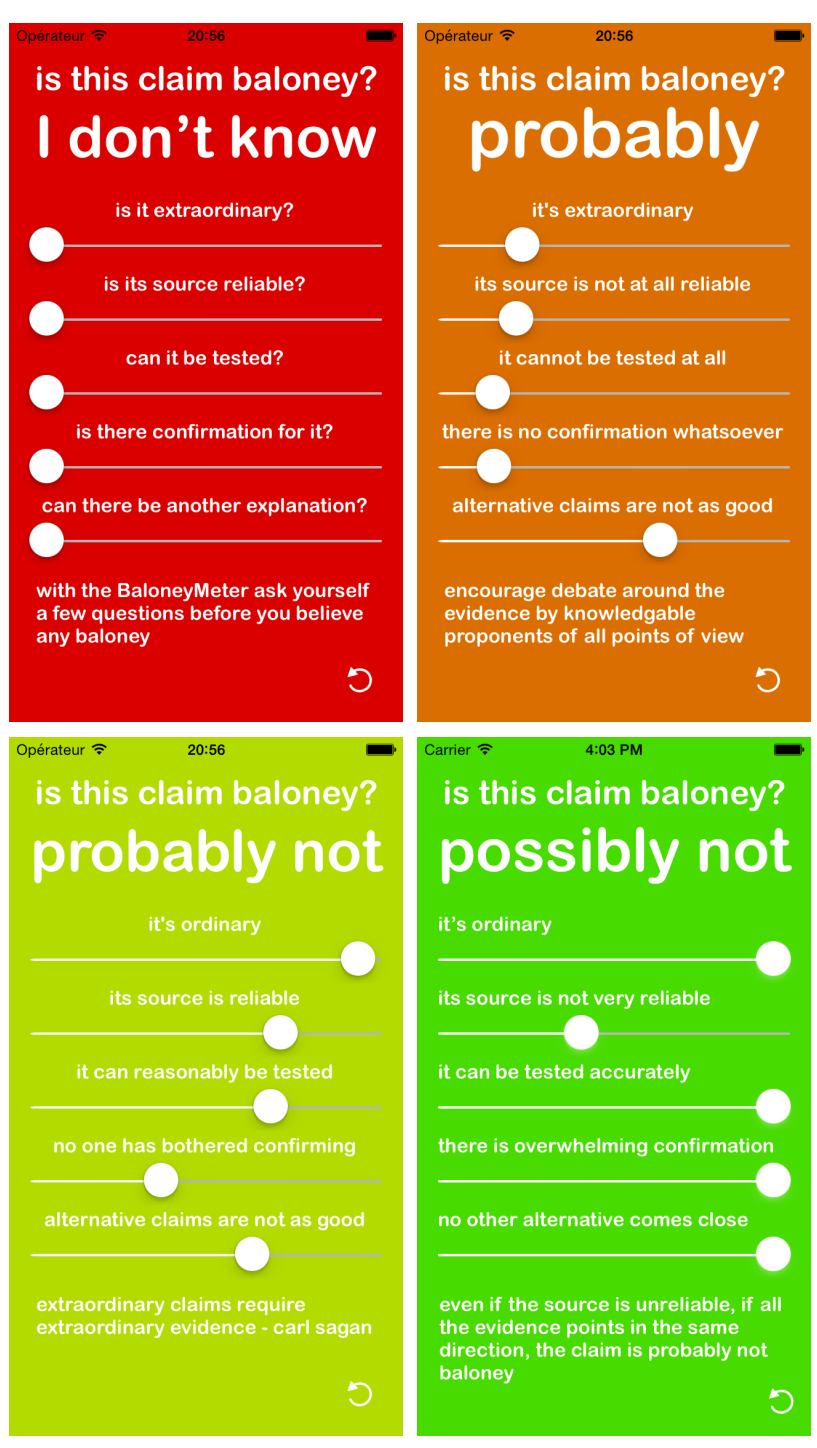

Figure 1: Screenshots of the BaloneyMeter app.
The user interface is minimalistic and only uses one screen. Users can move a slider for each question, answering it in a general way. Once all questions are answered, the app gives a general impression if the claim is baloney or not, with a tip at the bottom of the screen (e.g. remember that arguments from authority carry little weight, follow the evidence).

\section{Case study}

We performed a premliminary case study in a class of 150 first-year Bachelor engineering students during a course on

Global Issues in Communication at the École

Polytechnique Fédérale de Lausanne (EPFL) in

Switzerland. We designed a three hour-lecture on critical information assessment structured around the

BaloneyMeter questions and presented the application to students at the beginning of the first lecture and encouraged the students to play with it.

To get an idea of the level of paranormal belief of the class, we asked students to fill in the French version [3] of the Revised Paranormal Belief Scale [22] (RPBS). We obtained 140 replies (93\%). The RPBS has a total of 24 questions grouped in seven dimensions: traditional religious beliefs (e.g., belief in a God, an afterlife, Hell/Heaven), PSI (e.g., being able to move an object with the mind), Witchcraft (e.g., some people can cast spells on others), Superstition (e.g., 13 is an unlucky number), Spiritualism (e.g., the mind can travel oustide the body), Extraordinary Life Forms (e.g., the Loch Ness Monster), and Precognition (e.g., psychics can predict the future). For each question, there are 7 possible answers from $1-$ I totally disagree, to $7-$ I totally agree).

The results are presented in Figure 2 and agregate the different items per dimension. They convey the fact that 
the level of paranormal belief is overall very low in this sample. Except for Traditional Religious Beliefs,

Spiritualism and Precognition, most students (i.e., $>50 \%$ ) totally disagree with all claims and less than $10 \%$ even slighty agree with any of the claims. Traditional Religious Beliefs have the highest score, but they are still only held by a minority. Considering that some research suggests that paranormal beliefs are present in most of the population no matter the level of education [9], this sample might be especially skeptical of paranormal claims.

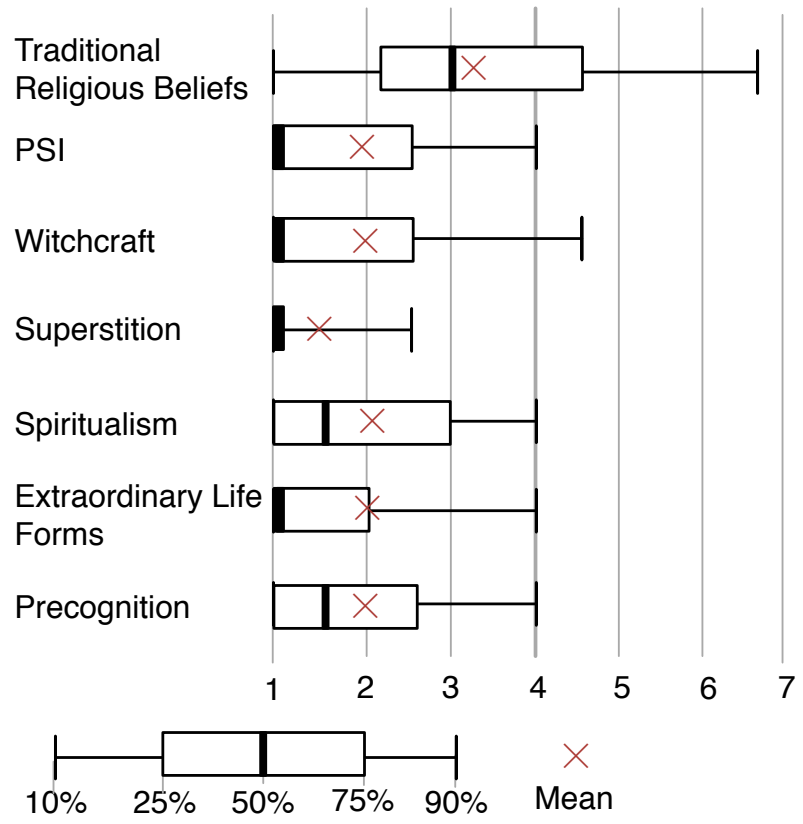

Figure 2: Results of the revised paranormal belief scale. The responses range from I totally disagree (1) to I totally Agree

(7). The outer vertical lines delimit the $90 \%$ of responses, the box delimits the 1 st and 3 rd quartiles. The median value and the mean are also shown. $\mathrm{N}=140$.

\section{Usability evaluation}

We performed a usability study of the BaloneyMeter using the System Usability Scale (SUS) and the AttrakDiff2 questionnaire after the course. We received 74 responses $(49 \%)$. We also asked a series of open questions on the improvements students would like to see in the app.

The AttrakDiff 2 results presented in Figure 3 show a generally positive attitude towards the app. Users find it especially clearly structured and simple, predictable and practical. Furthermore, they find it rather stylish, imaginative and good.

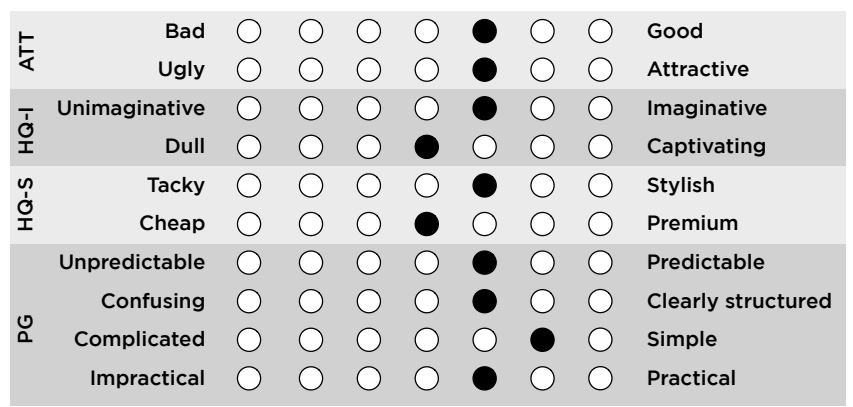

Figure 3: AttrakDiff results. The black dots represent the median. $\mathrm{N}=74$.

The mean of the 10 items of the SUS questionnaire was computed, achieving good reliability (Cronbach's $\alpha=0.85$ ). It resulted in a SUS score of 70 which means OK to Good usability. The shortcomings of the app were linked to usefulness rather than ease of use. For instance one student found that "the app is useless, it is made for people who cannot assess the reliability of information on their own!" A student also regreted that "the results only reflect one's own jugment" and then added that "it would be interesting to get data to confirm one's impressions." 


\section{Discussion and future work}

In this work, we have higlighted the importance and the difficulty of teaching critical thinking skills. Furthermore, we have noted the lack of research in the $\mathrm{HCl}$ literature on the subject. To address this research gap, we have designed a first version of the BaloneyMeter, a mobile application to augment face-to-face interaction in the classroom with a digital interface. In this version, the main function of the BaloneyMeter is to provide a playful way to access the main questions one should ask oneself before accepting a claim. Our positive usability results encourage us to push the mobile interaction further by answering the folowing research question:

RQ: How should blended mobile interaction be designed to increase critical thinking?

Potential hints to the answer to this question might come from designing features which allow users to provide self-generated arguments for certain claims, as research indicates that such an exercice positively favors founded vs unfounded beliefs [12]. Furthermore, as hinted in [8], allowing users to share their arguments with friends can potentially be more persuasive than opinions coming from, say, teachers. Finally, adding some gamification feature, with a sort of quizz for students, where they can test their skills might be valuable to improve user engagement as indicated by one of the participants quoted above.

To address this research question, we plan to design a full version of the BaloneyMeter containing such assessment and peer-to-peer gamification features and evaluate them in a classroom setting. In order to validate the design we will evaluate the usability of the app, but also learning outcomes, which will be measured in changes of beliefs and also in changes of critical assessment of information using a control group. For the change of beliefs we will use the RPBS, but we will augment it with more widely accepted beliefs around alternative medicine, similarly to the method employed in [9]. More specifically, in a first phase, we plan to conduct a between subject experimental design with two groups of participants. The first group will be required to fill in the augmented RPBS questionnaire without any prior intervention. The second group will be required to use the BaloneyMeter app to assess 5 claims contained in the augmented RPBS and then fill in the augmented RPBS questionnaire. This experiment will allow us to assess the impact of the BaloneyMeter on beliefs that have been assessed with it. Furthermore, it will also allow us to determine if its critical assessment spills over to other unfounded claims. In a second phase, we will perform a within subject experimental design. That is, we will teach the critical thinking course to all participants using the BaloneyMeter as support. After the course the participants will be required to fill in the questionnaire again, once directly after the course and once at the end of the semester. With this design, we will be able to assess if the course influences the beliefs of participants and if these changes are persistant.

We believe that answering this research question will not only allow to further the knowledge in $\mathrm{HCl}$, but addresses the more profound issue, which is to provide critical thinking skills to citizens to take informed decisions in a democracy.

\section{Acknowledgements}

We thank Bret Syfert for his work on the user interface and the app icon design. 


\section{References}

[1] Aarnio, K., and Lindeman, M. Paranormal beliefs, education, and thinking styles. Personality and Individual Differences 39, 7 (2005), 1227-1236.

[2] Barr, N., Pennycook, G., Stolz, J. A., and Fugelsang, J. A. The brain in your pocket: Evidence that smartphones are used to supplant thinking. Computers in Human Behavior 48 (2015), 473-480.

[3] Bouvet, R., Djeriouat, H., Goutaudier, N., Py, J., and Chabrol, H. Validation française de la revised paranormal belief scale. L'Encéphale 40, 4 (2014), 308-314.

[4] Brotherton, R., and Eser, S. Bored to fears: Boredom proneness, paranoia, and conspiracy theories. Personality and Individual Differences 80 (2015), 1-5.

[5] Brotherton, R., and French, C. C. Belief in conspiracy theories and susceptibility to the conjunction fallacy. Applied Cognitive Psychology 28, 2 (2014), 238-248.

[6] Darwin, H., Neave, N., and Holmes, J. Belief in conspiracy theories. the role of paranormal belief, paranoid ideation and schizotypy. Personality and Individual Differences 50, 8 (2011), 1289-1293.

[7] Gantz, J., and Reinsel, D. Extracting value from chaos. IDC iview, 1142 (2011), 9-10.

[8] Garrett, R. K. Troubling consequences of online political rumoring. Human Communication Research 37, 2 (2011), 255-274.

[9] Kane, M. J., Core, T. J., and Hunt, R. R. Bias versus bias: Harnessing hindsight to reveal paranormal belief change beyond demand characteristics. Psychonomic bulletin \& review 17, 2 (2010), 206-212.

[10] Lewandowsky, S., Ecker, U. K., Seifert, C. M., Schwarz, N., and Cook, J. Misinformation and its correction continued influence and successful debiasing. Psychological Science in the Public Interest 13, 3 (2012), 106-131.

[11] Mccaffree, K., and Saide, A. Why is critical thinking so hard to teach? In Skeptic Magazine, vol. 19 (2014), 54-57.

[12] Miller, R. L., and Wozniak, W. Counter-attitudinal advocacy: Effort vs. self-generation of arguments. Current Research in Social Psychology 6, 4 (2001), 46-57.

[13] Mirowsky, J., and Ross, C. E. Paranoia and the structure of powerlessness. American Sociological Review (1983), 228-239.

[14] Pennycook, G., Cheyne, J. A., Seli, P., Koehler, D. J., and Fugelsang, J. A. Analytic cognitive style predicts religious and paranormal belief. Cognition 123, 3 (2012), 335-346.

[15] Sagan, C. The fine art of baloney detection. Paranormal Claims: A Critical Analysis (2007), 1.

[16] Sagan, C. Demon-haunted world: science as a candle in the dark. Ballantine Books, 2011

[17] Shermer, M. Baloney detection. Scientific American 285, 5 (2001), 36.

[18] Shtulman, A. Epistemic similarities between students' scientific and supernatural beliefs. Journal of Educational Psychology 105, 1 (2013), 199.

[19] Stempel, C., Hargrove, T., and Stempel, G. H. Media use, social structure, and belief in $9 / 11$ conspiracy theories. Journalism \& Mass Communication Quarterly 84, 2 (2007), 353-372.

[20] Svanaes, D., and Verplank, W. In search of metaphors for tangible user intefaces. In Proceedings of DARE 2000 on Designing augmented reality environments, ACM (2000), 121-129. 
[21] Tennent, P., Reeves, S., Benford, S., Walker, B., Marshall, J., Brundell, P., Meese, R., and Harter, P. The machine in the ghost: augmenting broadcasting with biodata. In CHI'12 Extended Abstracts on Human Factors in Computing Systems, ACM (2012), 91-100.

[22] Tobacyk, J. J. A revised paranormal belief scale. The International Journal of Transpersonal Studies 23, 23 (2004), 94-98.
[23] Tsai, C.-Y., Lin, C.-N., Shih, W.-L., and Wu, P.-L. The effect of online argumentation upon students' pseudoscientific beliefs. Computers \& Education 80 (2015), 187-197.

[24] Willingham, D. T. Critical thinking: Why is it so hard to teach? Arts Education Policy Review 109, 4 (2008), 21-32. 